\title{
EXPERIMENTAL INVESTIGATION OF AN ACOUSTIC METAMATERIAL BARRIER DESIGN COMPOSED OF A SQUARE PRISM WITHIN A HEXAGONAL RECESS
}

\author{
Luo Mu ${ }^{1}$, Zamir Aimaduddin Zulkefli1* ${ }^{*}$, Nawal Aswan Abdul Jalil ${ }^{1}$, Azma Putra ${ }^{2}$, \\ Muhammad Nur Othman², and Raja Mohd Kamil Raja Ahmad ${ }^{3}$ \\ ${ }^{1}$ Department of Mechanical and Manufacturing Engineering, Faculty of Engineering, Universiti Putra \\ Malaysia, Selangor, Malaysia, e-mail: luomu9788@gmail.com, *zamirdin@upm.edu.my, \\ nawalaswan@upm.edu.my \\ ${ }^{2}$ Department Faculty of Mechanical Engineering, Universiti Teknikal Malaysia Melaka, Melaka, Malaysia, \\ e-mail: azma.putra@utem.edu.my,muhammadnur@utem.edu.my \\ ${ }^{3}$ Department of Electrical and Electronics Engineering, Faculty of Engineering, Universiti Putra Malaysia, \\ Selangor, Malaysia, e-mail: kamil@upm.edu.my
}

Received Date: July 31, 2020; Revised Date: December 7, 2020; Acceptance Date: March 8, 2021

\begin{abstract}
This paper presents an experimental investigation of an acoustic barrier composed out of an acoustic metamaterial unit cell. The design of the unit cell consists of a square prism, acting as a resonator, within a hexagonal recess manufactured out of a single material. Two materials were used to manufacture the unit cell: PolyMide Polycarbonate and Polylite Polylactic Acid. The acoustic performance of the unit cell was quantified for both materials using the acoustic absorption coefficient and acoustic transmission loss values for frequencies between $100 \mathrm{~Hz}$ and $5,000 \mathrm{~Hz}$. The experimental results indicate that the design reduced the peak absorption coefficient for both materials while also introducing two additional peaks at around $1,500 \mathrm{~Hz}$ and at $4,000 \mathrm{~Hz}$. Changes to the absorption coefficient values were observed for frequencies above $1,000 \mathrm{~Hz}$ while minimal changes were observed for frequencies below $1,000 \mathrm{~Hz}$. These results indicate that the proposed design, is able to widen the effective frequency band, or stop band for acoustic absorption for frequencies above $1,000 \mathrm{~Hz}$ compared to the absorption coefficient of the material. The experimental results also indicate that the design increases the peak transmission loss by about $7 \mathrm{~dB}$ at 4,000 $\mathrm{Hz}$. For sounds below $3,000 \mathrm{~Hz}$, the design will only change the transmission loss by about $3 \mathrm{~dB}$ for frequencies between $100 \mathrm{~Hz}$ to $3,000 \mathrm{~Hz}$. These results indicate that the acoustic metamaterial design, consisting of resonator in a recess manufactured out of a single material, is able to broaden the effective frequency range for sound absorption for frequencies between $1,000 \mathrm{~Hz}$ and $4,000 \mathrm{~Hz}$ and at increasing the transmission loss values for frequencies between $3,000 \mathrm{~Hz}$ and $5,000 \mathrm{~Hz}$. It can be concluded that the resonator in recess metamaterial design, manufactured out of a single material, can be used to increase the stop band for acoustic absorption for frequencies above $1,000 \mathrm{~Hz}$ and to increase the transmission loss for frequencies above $3,000 \mathrm{~Hz}$.
\end{abstract}

Keywords: Acoustic barrier design, Acoustic metamaterial, Noise control

\section{Introduction}

\section{Background}

The worldwide increases in vehicular traffic have led to an increase in acoustic pollution, especially within an urban environment. To alleviate the impact of acoustic pollution, various noise mitigation efforts are currently being taken. One approach is to utilize acoustic 
barriers to reduce the transmission of noise from highways and roads to the surrounding geographical areas. The effectiveness of acoustic barriers is typically increased by increasing the overall density, mass, or thickness of said barriers. This approach to increasing the effectiveness of acoustic barriers however, brings additional weight and cost requirements, as well as limiting the ability of placing acoustic barriers only in areas with the necessary space.

Over the past few years, novel approaches to increasing the effectiveness of acoustic barriers have been developed based on the unique characteristics of acoustic metamaterials. Acoustic metamaterials are engineered materials that can influence acoustic waves beyond what can be achieved using naturally-occurring materials [1]. The unique characteristics of metamaterials derive from its structure, rather than from its material makeup meaning that acoustic metamaterials can be designed to target specific acoustic issues. Acoustic metamaterials, in the form of locally resonant materials, have also shown great potential in improving the effectiveness of noise mitigation efforts, such as increasing the acoustic performance of acoustic barriers without corresponding increases in density, mass, or thickness [2].

This study experimentally investigates the effect of an acoustic metamaterial design on the performance of an acoustic barrier design. The acoustic metamaterial unit cell consists of a hexagonal recess containing a square prism acting as a resonator. More specifically, this study examines the impact of the acoustic metamaterial design, especially the resonator size on the acoustic performance of the design. The acoustic performance was quantified through two metrics: the sound absorption coefficient and transmission loss values.

This paper is organized as follows. A brief background of acoustic metamaterials is presented. This is followed by a discussion of the design and material properties of the acoustic metamaterial unit cell and a discussion of the experimental setup. The results are then discussed and main conclusions summarized.

\section{Noise Mitigation Using Acoustic Metamaterials}

Acoustic metamaterials, are materials with acoustic performance determined primarily by its internal structure as opposed to its chemical composition or material makeup. Because of this, metamaterials can be designed to have macroscopic properties not available in naturally occurring materials, such as negative density and negative stiffness. These properties allow the acoustic metamaterials the ability to strongly interact with acoustic waves, potentially impeding its transmission, focusing it, or refracting it away from its original direction. The focus on acoustic metamaterials are due to the potential advantage of the metamaterial design in overcoming limitations of bulky structures in addressing noise issues in the audible frequency regime [3]. This potential advantage comes about due to its ability to provide acoustical benefits with minimum weight penalty [4] and the ability to effectively address complex noise issues [5] through geometrical manipulation and repetition of internal structures [6]. This study therefore utilizes an acoustic metamaterial design approach to design an acoustic barrier for potential use as noise barriers in roadside applications, where the minimum weight penalty and ability to address complex noise issues through geometric manipulation would be very advantageous to addressing noise issues.

Acoustic metamaterials exploit the mechanical and acoustical resonances in its structure in order to strongly interact with the incoming acoustic waves at particular frequencies $[7,8]$. The destructive interference between the incoming acoustic waves with the re-radiated waves from local resonators, results in a reduced transmission of the original acoustic signal. This effect occurs at particular frequencies and over particular frequency range, called the stop band range. This type of acoustic interference is referred to as Fano- 
type interference [9]. Combining different acoustic metamaterial designs with different stop band ranges can result in acoustic barriers with multiple stop band ranges $[10,11]$ that can be utilized to address specific acoustic issues.

Various metamaterial designs have been proposed. For example, metal spheres can be incorporated in soft silicone epoxy to exhibit negative dynamic mass and strong elastic wave attenuation [12]. Meanwhile membrane-type metamaterial uses concentrated mass attached to a membrane to behave as a spring-mass-damper system. These spring-massdamper systems generate sub-wavelength sound transmission [13,14] and absorption [15]. Other design approach consists of an open duct incorporating coiled resonators for low frequency sound transmission loss within a compact design [4]. More recently, a design consisting of a metal helix structure perforating a viscoelastic damping rubber shows excellent acoustic absorption performance for frequencies below 1,000 Hz [16]. Similar approaches utilizing mass-in-mass lattice model, exhibiting multiple stop band behavior, have been proposed [17].

For industrial and practical applications, the designs need to not only meet acoustic requirements, but to take into account the manufacturability of the designs. Manufacturing issues have been shown to arise even if a small-scale design was considered [18]. These scaling issues can be minimized by utilizing simple designs such plate-type acoustic metamaterials with internal resonators. This plate-type metamaterial is more easily manufactured while still achieving customizable acoustical performance [19]. Manufacturing issues can also be minimized by utilizing designs made out of a single material, reducing manufacturing complications. Acceptable acoustic performance over specific frequency ranges have been shown to be achievable through the use of concave starshaped structures manufactured out of only a single material [20]. Significant noise reduction capabilities can also be achieved through the use of combined heterogeneous double-split hollow sphere which are also simple to manufacture [21]. High frequency noise reduction has also been shown to be achievable using gypsum-based 3D printing technology [22]. The approach taken in this study was to take into account the manufacturability of the acoustic barrier in the design process by utilizing a simple metamaterial design and manufacturing out of a single material using 3D printing technology. The results of this study are expected to be used to develop simple metamaterial design suitable for mass production via 3D printing technology while still able to address specific noise issues.

\section{Material and Methods}

\section{Design and Material Selection}

The acoustic metamaterial design used for the unit cell consists of a square prism, acting as a resonator, placed longitudinally within a hexagonal recess. The design was manufactured as one solid piece out of a single material using a 3D printing manufacturing process. Furthermore, the size of the resonator was varied to determine the effect of resonator size on the acoustic performance of the design.

Table 1 shows the dimensions and design parameters of the unit cells that were tested. The unit cells were manufactured out of two materials: PolyMide Polycarbonate (CoPA) and Polylite Polylactic Acid (PLA). PolyMide CoPA is a carbon fiber reinforced nylon while PLA is a thermoplastic polyester. Both materials were chosen based on its use in the 3D printing manufacturing process. The material properties of PolyMide CoPA are: Young's Modulus value of 2,223 $\pm 199 \mathrm{MPa}$, a Tensile Strength value of $66.2 \pm 0.9 \mathrm{MPa}$, a Bending Modulus of $8,339 \pm 369 \mathrm{MPa}$, and a density at $21.5^{\circ} \mathrm{C}$ of $1.12 \mathrm{~g} / \mathrm{cm}^{3}$ [23] 
Meanwhile the material properties of PLA are: Young's Modulus value of 2,636 $\pm 330 \mathrm{MPa}$, a Tensile Strength value of 46.6 $\pm 0.9 \mathrm{MPa}$, a Bending Modulus of 3,283 $\pm 132 \mathrm{MPa}$, and a density at $21.5^{\circ} \mathrm{C}$ of $1.21 \mathrm{~g} / \mathrm{cm}^{3}[24]$.

Table 1. Dimensional and Design Parameters of Unit Cell Design Samples

\begin{tabular}{|c|c|c|c|c|c|}
\hline No. & Identifier & Description & $\begin{array}{l}\text { Dimensions } \\
\text { of Unit Cell } \\
(\mathrm{mm})\end{array}$ & $\begin{array}{c}\text { Dimensions } \\
\text { of Hexagonal } \\
\text { Recess (mm) }\end{array}$ & $\begin{array}{c}\text { Dimensions } \\
\text { of Resonator } \\
(\mathrm{mm})\end{array}$ \\
\hline 1. & Solid & Solid circular prism & $\begin{array}{l}\mathrm{D}_{\mathrm{c}}: 33.3 \mathrm{~mm} \\
\mathrm{H}_{\mathrm{c}}: 30.0 \mathrm{~mm}\end{array}$ & None & None \\
\hline 2. & $\begin{array}{c}\text { Small } \\
\text { Resonator }\end{array}$ & $\begin{array}{c}\text { Square resonator } \\
\text { within a hexagonal } \\
\text { recess }\end{array}$ & $\begin{array}{l}\mathrm{D}_{\mathrm{c}}: 33.3 \mathrm{~mm} \\
\mathrm{H}_{\mathrm{c}}: 30.0 \mathrm{~mm}\end{array}$ & $\begin{array}{l}\mathrm{H}_{\mathrm{h}}: 20.0 \mathrm{~mm} \\
\mathrm{~L}_{\mathrm{h}}: 10.0 \mathrm{~mm}\end{array}$ & $\begin{array}{l}\mathrm{H}_{\mathrm{r}}: 8.4 \mathrm{~mm} \\
\mathrm{~L}_{\mathrm{r}}: 1.8 \mathrm{~mm}\end{array}$ \\
\hline 3. & $\begin{array}{l}\text { Medium } \\
\text { Resonator }\end{array}$ & $\begin{array}{c}\text { Square resonator } \\
\text { within a hexagonal } \\
\text { recess }\end{array}$ & $\begin{array}{l}\mathrm{D}_{\mathrm{c}}: 33.3 \mathrm{~mm} \\
\mathrm{H}_{\mathrm{c}}: 30.0 \mathrm{~mm}\end{array}$ & $\begin{array}{l}\mathrm{H}_{\mathrm{h}}: 20.0 \mathrm{~mm} \\
\mathrm{~L}_{\mathrm{h}}: 10.0 \mathrm{~mm}\end{array}$ & $\begin{array}{c}\mathrm{H}_{\mathrm{r}}: 11.2 \mathrm{~mm} \\
\mathrm{~L}_{\mathrm{r}}: 2.4 \mathrm{~mm}\end{array}$ \\
\hline 4. & $\begin{array}{c}\text { Large } \\
\text { Resonator }\end{array}$ & $\begin{array}{c}\text { Square resonator } \\
\text { within a hexagonal } \\
\text { recess }\end{array}$ & $\begin{array}{l}\mathrm{D}_{\mathrm{c}}: 33.3 \mathrm{~mm} \\
\mathrm{H}_{\mathrm{c}}: 30.0 \mathrm{~mm}\end{array}$ & $\begin{array}{l}\mathrm{H}_{\mathrm{h}}: 20.0 \mathrm{~mm} \\
\mathrm{~L}_{\mathrm{h}}: 10.0 \mathrm{~mm}\end{array}$ & $\begin{array}{c}\mathrm{H}_{\mathrm{r}}: 14.0 \mathrm{~mm} \\
\mathrm{~L}_{\mathrm{r}}: 3.0 \mathrm{~mm}\end{array}$ \\
\hline
\end{tabular}
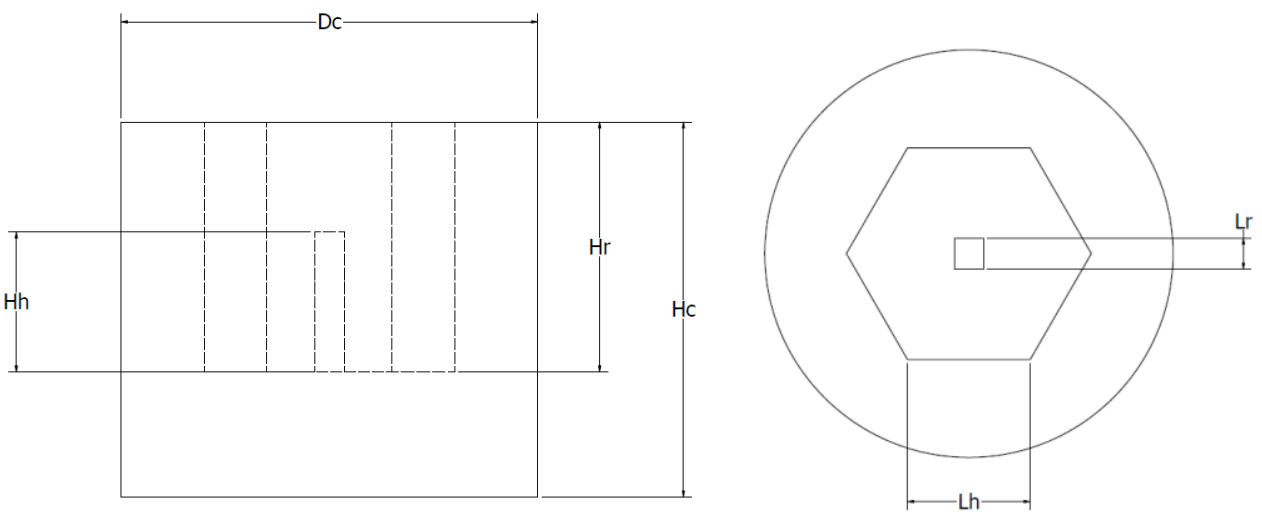

Figure 1. Engineering drawings of unit cell design with dimensional parameters

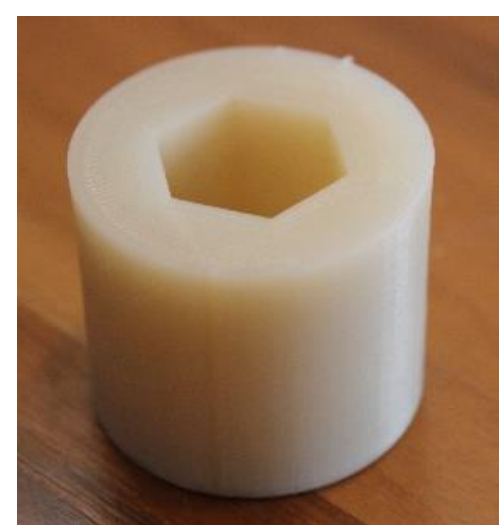

(a)

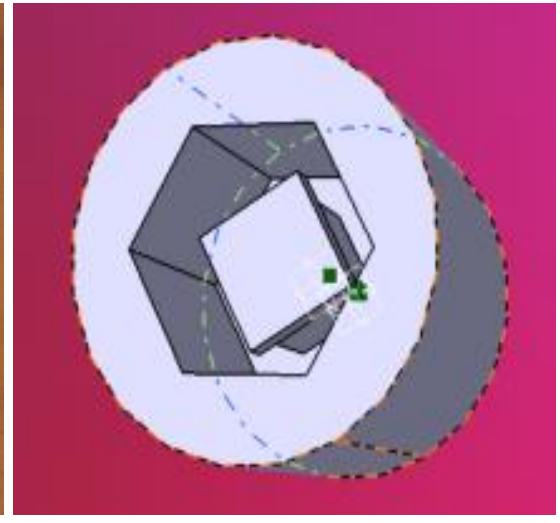

(b)

Figure 2. (a) Photograph of unit cell as manufactured and (b) isometric 3D view of unit cell 
The design of sample No. 1 consist of a solid circular prism, used to determine the baseline absorption coefficient and transmission loss values of the materials. The design of unit cell No. 2, No. 3, and No. 4 consist of a square prism, acting as the resonator, centrally located within the hexagonal recess. Design No. 2 has the smallest resonator size, with the size increasing for design No. 3 and No. 4. Figure 1 shows the engineering drawings of the designs consisting of a square prism in a hexagonal recess. Figure 1 also indicates the unit cell's dimensional parameters, whose values for the different cell designs are given in Table 1. Figure 2(a) shows a photograph of the unit cell as manufactured while Figure 2(b) shows an isometric 3D view of the unit Experimental Set-Up

The acoustic characteristics of the unit cell design, specifically the acoustic absorption coefficient and transmission loss, were determined using an impedance tube. These two values were selected as parameters to quickly assess the noise control impact of the designs especially in noise barrier applications. As highlighted in [25], the acoustic absorption coefficient is a parameter used to assess the basic acoustic characteristic of a material, including materials typically used as noise barriers. Simply put, the acoustic absorption coefficient measures the ability of the material to reflect the incoming acoustic energy. The acoustic absorption coefficient was therefore measured in order to assess the acoustic characteristic of the design in its potential role a noise barrier especially its reflective ability. The selection of the transmission loss values was in line with [2] in assessing the effectiveness of an acoustic metamaterial design in noise barrier applications. Simple put the transmission loss measure the ability to allow the acoustic energy to pass through the material, an important consideration for a potential noise barrier. Together these two values are expected to provide the needed information to determine the acoustic characteristics of the metamaterial design in its potential role as a noise barrier.

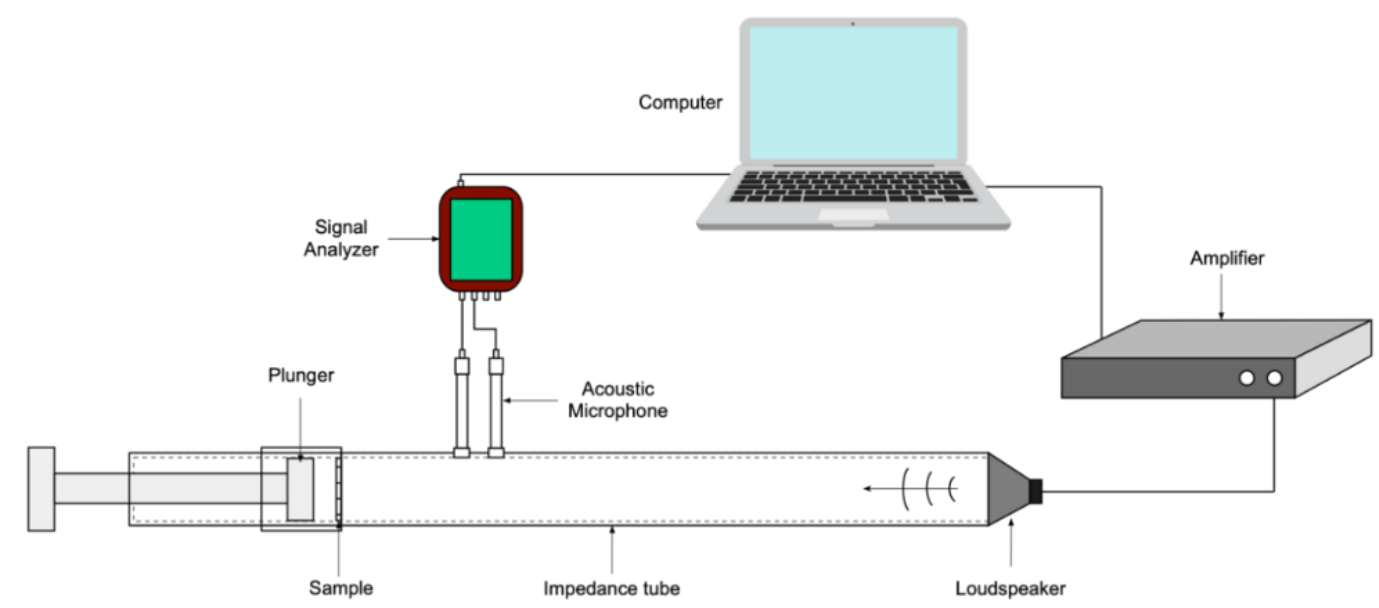

Figure 3. Schematic of setup to measure the absorption coefficient as per ASTM E1050 Standard

The experimental setup to measure the absorption coefficient was carried out according to ASTM E1050 standard [26]. The schematic of the experimental setup is shown in Figure 3 and additional parameters used in the experimental setup are given in Table 2. As shown in Figure 3, a two-microphone transfer function method was utilized to measure the absorption coefficient. Part of the incident acoustic signal from the loudspeaker was absorbed by the unit cell and the rest reflected. The difference between the incident and reflected signals, measured using acoustic microphones, was used to determine the absorption coefficient of the samples. 
Table 2. Parameters Used During the Measurement of the Absorption Coefficient

\begin{tabular}{|c|c|c|}
\hline No. & Parameter & Value \\
\hline 1. & Ambient temperature & $25.40{ }^{\circ} \mathrm{C}$ \\
\hline 2. & Distance of nearest microphone to sample & $50.80 \mathrm{~mm}$ \\
\hline 3. & Microphone spacing & $29.21 \mathrm{~mm}$ \\
\hline 4. & Number of lines & 801.00 \\
\hline 5. & Frequency resolution & $6.87 \mathrm{~Hz}$ \\
\hline 6. & Data acquisition hardware & Photon analyzer \\
\hline 7. & Speaker amplifier & Mudacity 1.3 \\
\hline 8. & Post-processing software & $0-5,000 \mathrm{~Hz}$ \\
\hline 9. & Frequency range & GRAS acoustic microphone type \\
\hline 10. & Microphone & 60 seconds \\
\hline 11. & & \\
\hline
\end{tabular}

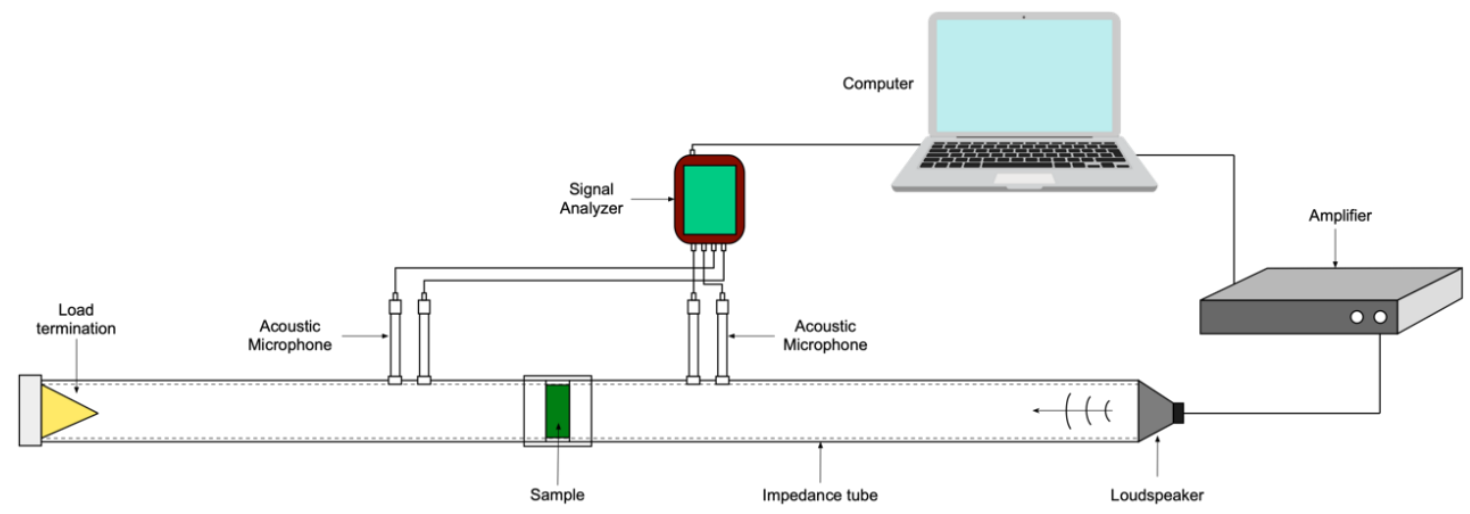

Figure 4. Schematic of setup to measure the transmission loss

Table 3. Parameters Used During the Measurement of the Transmission Loss

\begin{tabular}{|c|c|c|}
\hline No. & Parameter & Value \\
\hline 1. & Ambient temperature & $29.21{ }^{\circ} \mathrm{C}$ \\
\hline 2. & Distance of microphone 2 to sample & $50.80 \mathrm{~mm}$ \\
\hline 3. & Distance of microphone 3 to sample & $177.80 \mathrm{~mm}$ \\
\hline 3. & Microphone spacing [1-2] S1 & $29.21 \mathrm{~mm}$ \\
\hline 4. & Microphone spacing [3-4] S2 & $29.21 \mathrm{~mm}$ \\
\hline 4. & Number of lines & 801.00 \\
\hline 5. & Frequency resolution & $6.87 \mathrm{~Hz}$ \\
\hline 6. & Data acquisition hardware & Photon analyzer \\
\hline 7. & Speaker amplifier & MAdacity 1.3 \\
\hline 8. & Post-processing software & $0-5,000 \mathrm{~Hz}$ \\
\hline 9. & Frequency range of interest & GRAS acoustic microphone \\
\hline 10. & Microphone $46 \mathrm{~A}$ \\
\hline 11. & & 60 seconds \\
\hline
\end{tabular}

The measurements of the transmission loss values were carried out via the twoload method [27] a schematic of which is shown in Figure 4. The loud speaker generates a broadband random sound over the frequency range of interest, in this case $100 \mathrm{~Hz}$ to 5,000 
Hz. The difference between the incident signal and the transmitted signal, measured by the respective microphones, was used to determine the transmission loss of the sample. Additional parameters for the transmission loss measurement are given in Table 3.

\section{Results and Discussion}

\section{Acoustic Performance of Materials}

Figure 5 shows the measured absorption coefficient for the solid circular prism over the frequency range of $100 \mathrm{~Hz}$ to $5,000 \mathrm{~Hz}$. The results show that for both materials, PolyMide CoPA and PLA, the absorption coefficient shows similar general trends. Local peaks are observed at $160 \mathrm{~Hz}$ and at $800 \mathrm{~Hz}$. Maximum absorption coefficient values are also observed for both materials at $2,500 \mathrm{~Hz}$. Both materials also show local minimums at $1,000 \mathrm{~Hz}$ and at $4,000 \mathrm{~Hz}$. Below a frequency of $1,000 \mathrm{~Hz}$, both materials exhibit similar absorption coefficient performance except at $100 \mathrm{~Hz}$ when PLA shows a higher absorption coefficient value of 0.10 when compared to PolyMide CoPA absorption coefficient value of 0.00 . The measured absorption coefficient value of 0.00 for PolyMide CoPA, within limits of experimental errors, is in line with values of other reflective materials such as concrete, brick and plaster at low frequencies [28].

Differences are observed in the absorption coefficient values for frequencies above 1,000 Hz. For frequencies between 1,000 Hz and 1,600 Hz, PolyMide CoPA shows higher absorption coefficient values than PLA. Above $1,700 \mathrm{~Hz}$ however, PLA shows higher absorption coefficient values instead. PLA's absorption coefficient values reached a maximum of 0.59 at $2,500 \mathrm{~Hz}$ while PolyMide CoPA's maximum value is only 0.32 at the same frequency. At $4,000 \mathrm{~Hz}$ the absorption coefficient values of PLA is 0.05 while the absorption coefficient values of PolyMide CoPA is lower at around 0.00 . At $5,000 \mathrm{~Hz}$ PLA again shows a higher absorption coefficient value of 0.32 versus PolyMide CoPA value of 0.14 .

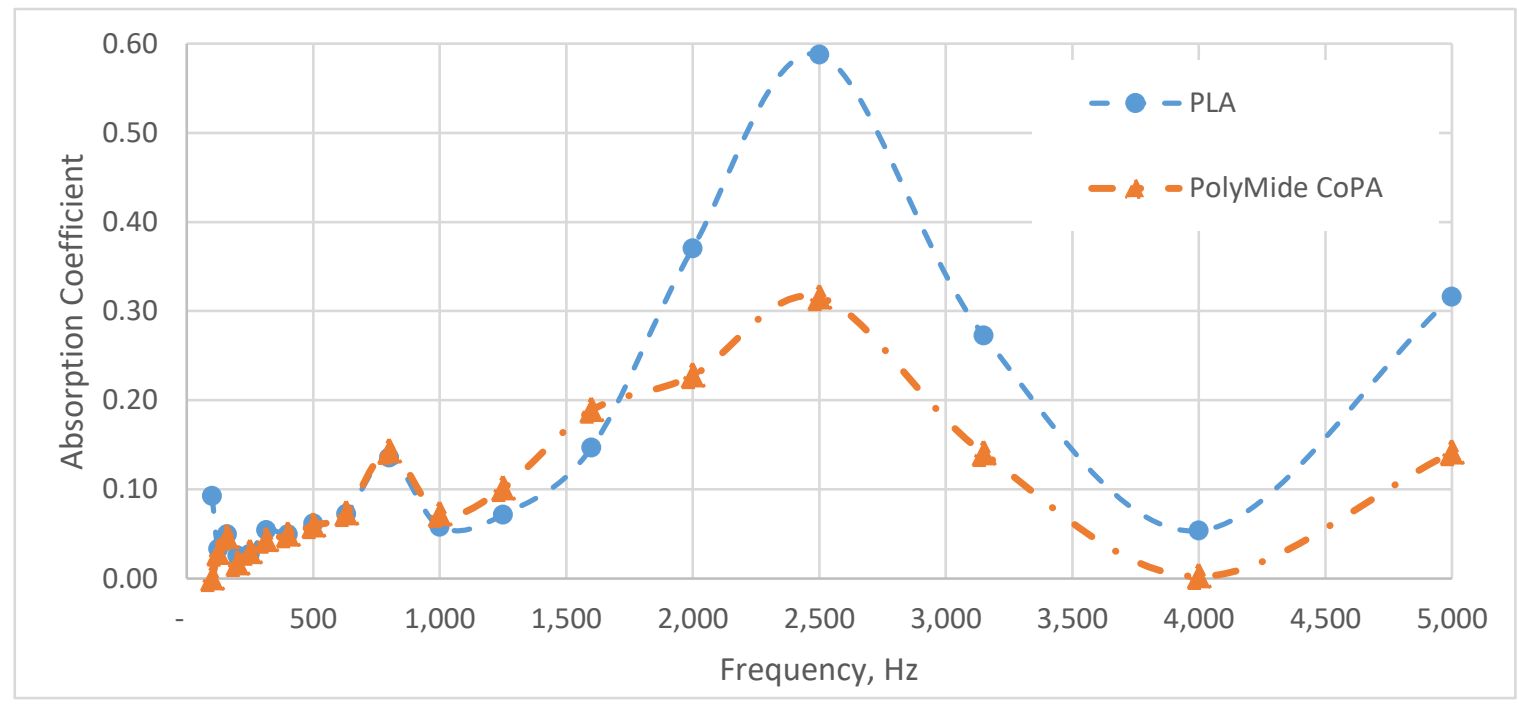

Figure 5. Absorption coefficient for solid circular prism

Figure 6 shows the measured transmission loss for the solid circular prism. The transmission loss value shows similar general performances for both materials gradually increasing to a maximum at $2,500 \mathrm{~Hz}$ and then gradually decreasing again. It is also observed that the transmission values for PolyMide CoPA were consistently higher (by around $10 \mathrm{~dB}$ ) 
than the transmission values of PLA over the frequency range. The transmission values of PLA however, does show a faster reduction especially between $3,000 \mathrm{~Hz}-4,000 \mathrm{~Hz}$ when compared to PolyMide CoPA. At 4,000 Hz, PLA reaches a constant transmission loss value of $14.2 \mathrm{~dB}$. At $200 \mathrm{~Hz}$, PolyMide CoPA shows a very small local peak of $15.4 \mathrm{~dB}$. For frequencies below $160 \mathrm{~Hz}$, both materials show similar transmission loss values.

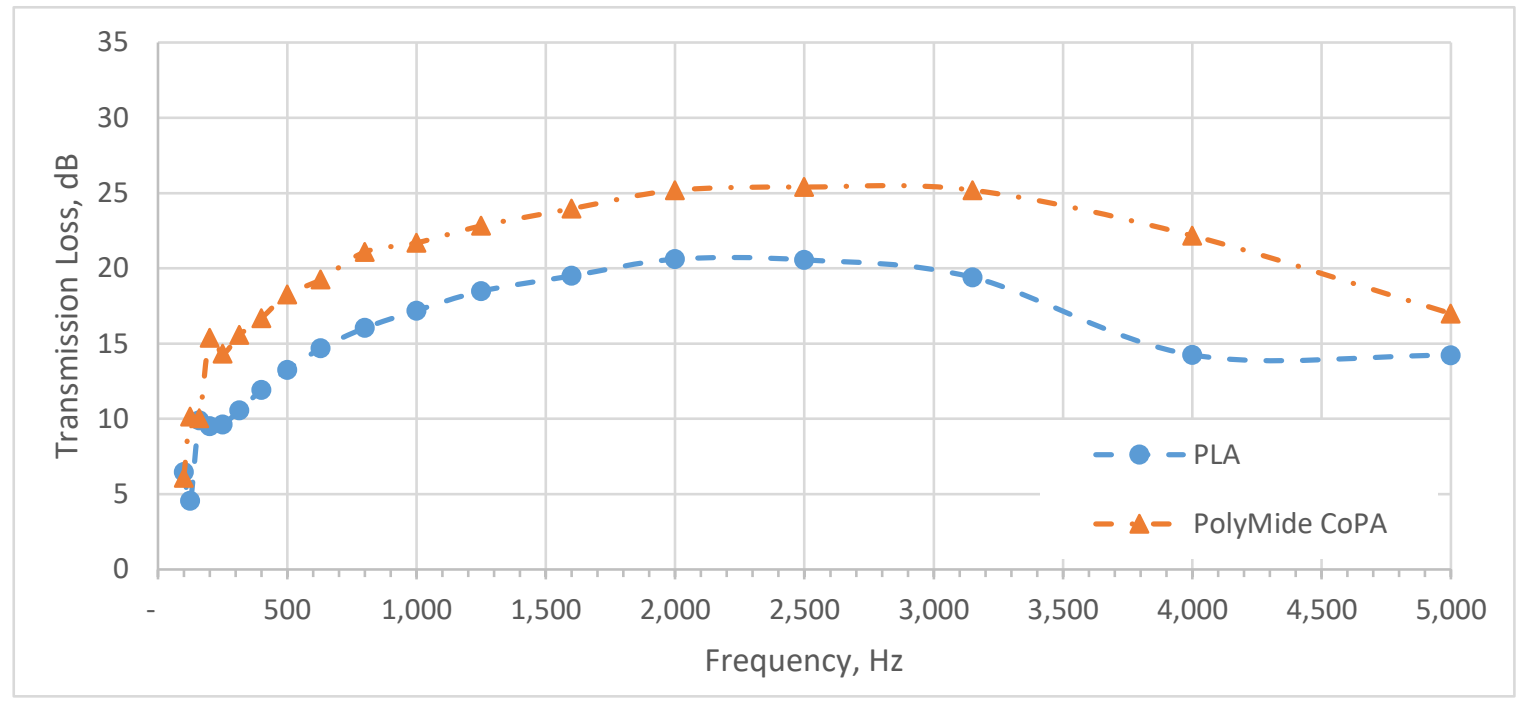

Figure 6. Transmission loss for solid circular prism

The results from Figures 5 and 6 indicate that PLA has higher sound absorbing capabilities especially for frequencies above $1,700 \mathrm{~Hz}$ when compared to PolyMide CoPA. One the other hand, PolyMide CoPA has consistently higher transmission loss capabilities than PLA for frequencies above $160 \mathrm{~Hz}$.

\section{Acoustic Performance of Metamaterial Designs}

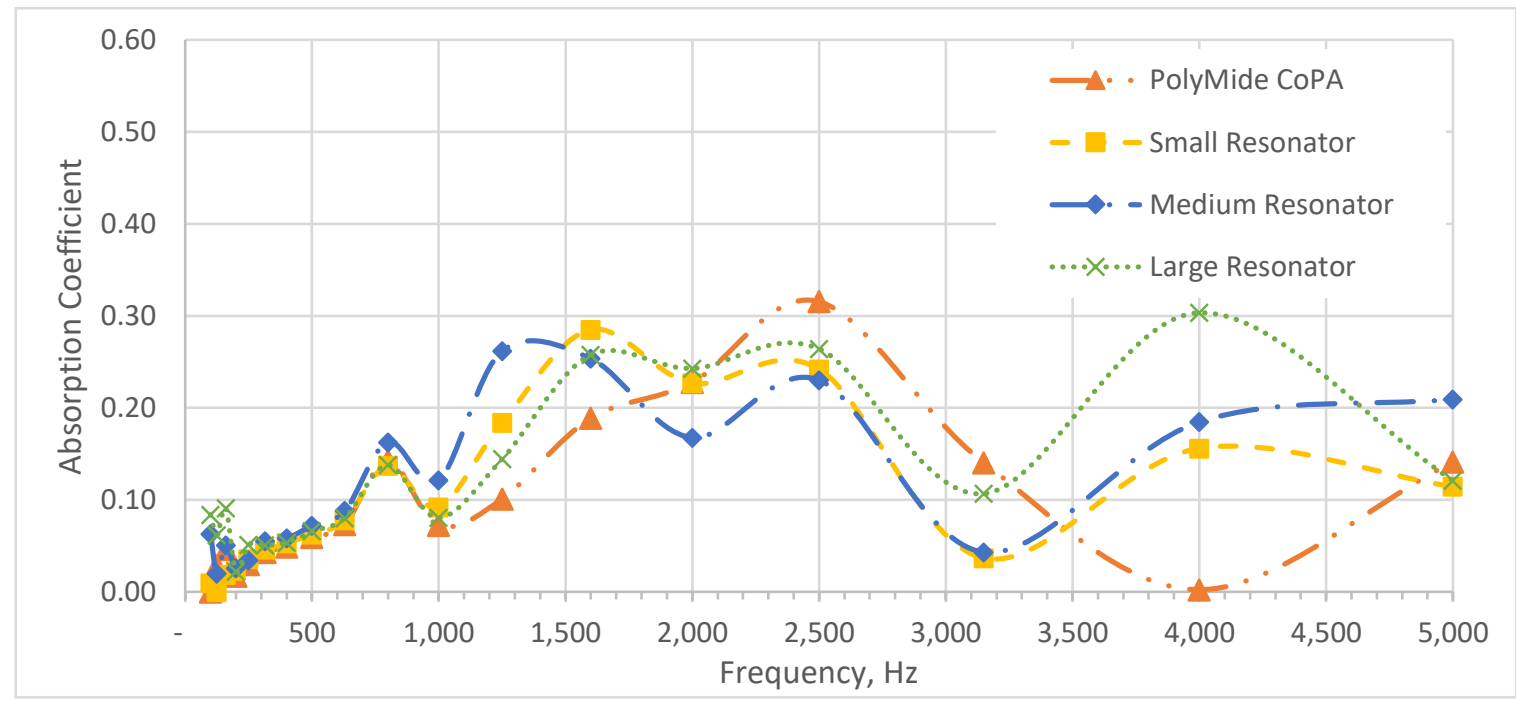

Figure 7. Absorption coefficient for unit cell designs made out of PolyMide CoPA

Figure 7 shows the measured absorption coefficient for all unit cell designs manufactured out of PolyMide CoPA over the frequency range of $100 \mathrm{~Hz}$ to $5,000 \mathrm{~Hz}$. The results show that the introduction of the resonator within a hexagonal recess changed the absorption 
coefficient values especially for frequencies above $800 \mathrm{~Hz}$. More generally, the introduction of the resonators and recess introduces localized peaks within the $1,000 \mathrm{~Hz}-2,000 \mathrm{~Hz}$ range and within the $3,500 \mathrm{~Hz}-4,500 \mathrm{~Hz}$ frequency range. Within the $1,000 \mathrm{~Hz}-2,000 \mathrm{~Hz}$ frequency range, the peaks varied between 0.25 and 0.28 in values. Within the $3,500 \mathrm{~Hz}-$ $4,500 \mathrm{~Hz}$ range, the additional peaks varied between 0.15 and 0.30 . Meanwhile the peak within the $2,000 \mathrm{~Hz}-3,000 \mathrm{~Hz}$ range was reduced from 0.30 to around $0.23-0.26$ when the resonator and recess was introduced into the design.

As the resonator size was increased the peaks within the $1,000 \mathrm{~Hz}-2,000 \mathrm{~Hz}$ range were not significantly affected. The peaks within the $3,500 \mathrm{~Hz}-4,500 \mathrm{~Hz}$ range however increased as the size of the resonators were increased from 0.16 for the small resonator to 0.30 for the large resonator. This increase was more significant as the resonator size was changed from medium to large when compared to the increase from small to medium. Finally, the peak within the $2,000 \mathrm{~Hz}-3,000 \mathrm{~Hz}$ range was also not significantly affected by the increase in the resonator size.

Figure 8 shows the measured absorption coefficient for all unit cell designs manufactured out of PLA over the frequency range of $100 \mathrm{~Hz}$ to $5,000 \mathrm{~Hz}$. The results show that the introduction of the resonator within a hexagonal recess changed the absorption coefficient values especially for frequencies above $800 \mathrm{~Hz}$. More generally, the introduction of the resonators introduces localized peaks within the $1,000 \mathrm{~Hz}-2,000 \mathrm{~Hz}$ range. These peaks were around $0.23-0.31$ in values. Similarly, within the $3,500 \mathrm{~Hz}-4,500 \mathrm{~Hz}$ range, additional peaks, with values ranging from 0.15 to 0.30 were introduced. Meanwhile the peak within the $2,000 \mathrm{~Hz}-3,000 \mathrm{~Hz}$ range was reduced from 0.59 to around $0.25-0.28$ when the resonator and recess was introduced into the design.

As the resonator size was increased, the peaks within the $1,000 \mathrm{~Hz}-2,000 \mathrm{~Hz}$ range were reduced from 0.31 to 0.23 . This reduction was more significant as the resonator sized was increased from small to medium compared to when the resonator size was increased from medium to large. The peaks within the $3,500 \mathrm{~Hz}-4,500 \mathrm{~Hz}$ range meanwhile increased as the size of the resonators were increased from 0.15 for the small resonator to 0.30 for the large resonator. This increase was more significant as the resonator size was changed from medium to large when compared to the increase from small to medium. Finally, the peak within the $2,000 \mathrm{~Hz}-3,000 \mathrm{~Hz}$ range was not significantly affected by the increase in the resonator size staying around $0.25-0.28$.

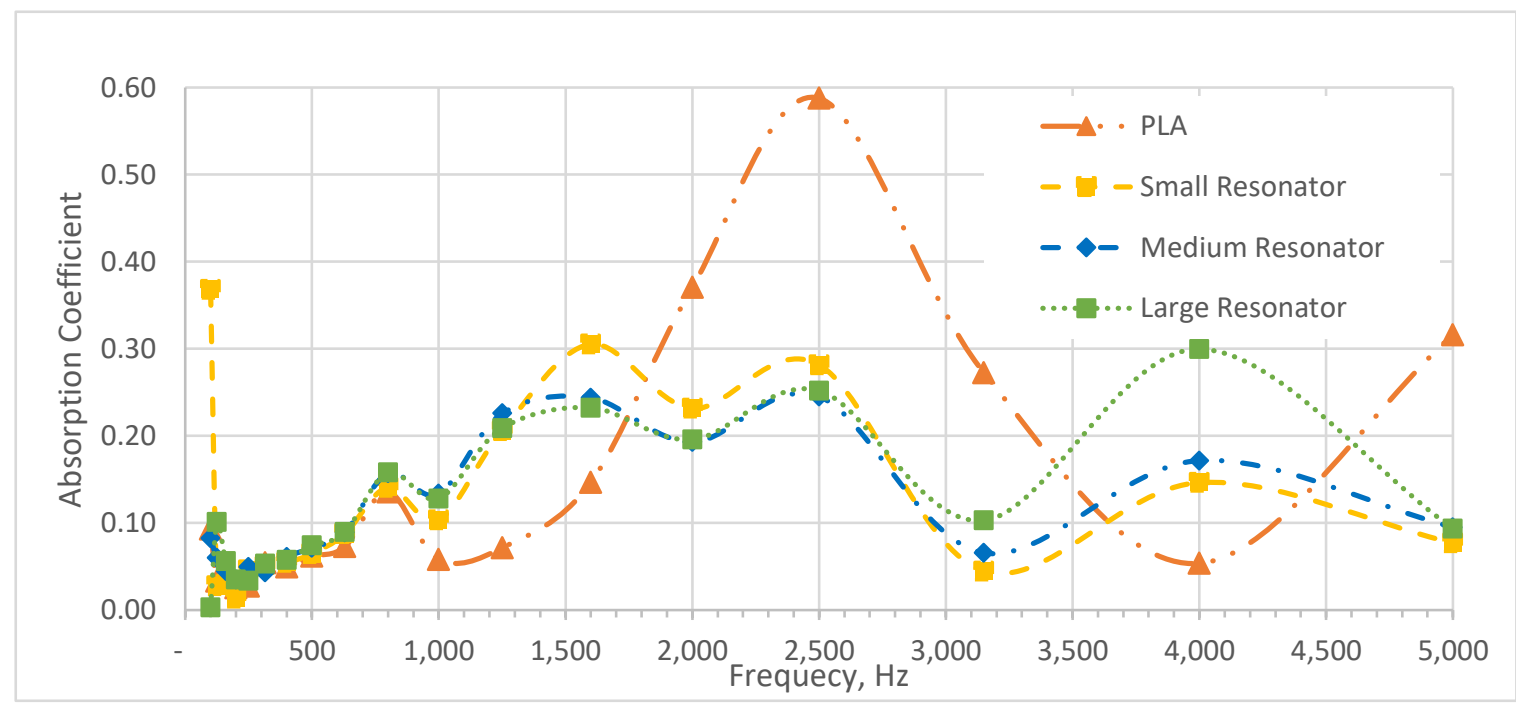

Figure 8. Absorption coefficient for unit cell designs made out of PLA 
The results in Figures 7 and 8 indicates that the introduction of a resonator within a hexagonal recess introduces local peaks within the $1,000 \mathrm{~Hz}-2,000 \mathrm{~Hz}$ and $3,500 \mathrm{~Hz}-$ $4,500 \mathrm{~Hz}$ frequency range. The introduction of the resonator in the design also reduces any peaks within $2,000 \mathrm{~Hz}-3,000 \mathrm{~Hz}$ to a value of around $0.25-0.30$. Furthermore, the peak within the $1,000 \mathrm{~Hz}$ to $3,000 \mathrm{~Hz}$ was less sensitive to changes in the resonator size. Meanwhile, the peak within the $3,500 \mathrm{~Hz}-4,500 \mathrm{~Hz}$ was more sensitive to changes in the resonator size. This indicates that the metamaterial design expands the effective frequency range, or stop band, of the absorption coefficient, through the introduction of additional peaks. The expansion is only observable for frequencies above $1,000 \mathrm{~Hz}$.

Figure 9 shows the transmission loss values of all unit cell designs manufactured out of PolyMide CoPA. The results show that a local minimum, with values in the $8 \mathrm{~dB}-10$ $\mathrm{dB}$ range was introduced for the small and large resonators at around $400 \mathrm{~Hz}$. In addition, a local peak, with values of around $26 \mathrm{~dB}-31 \mathrm{~dB}$, was introduced in the $3,500 \mathrm{~Hz}-4,500 \mathrm{~Hz}$ frequency range. Finally, for frequencies between $500 \mathrm{~Hz}-2,000 \mathrm{~Hz}$ the introduction of a resonator and recess reduces the transmission loss values when compared to the solid circular prism. The transmission loss values were higher for the medium and larger resonators for frequencies above $2,000 \mathrm{~Hz}$, while it was only higher for the small resonator for frequencies above $3,400 \mathrm{~Hz}$.

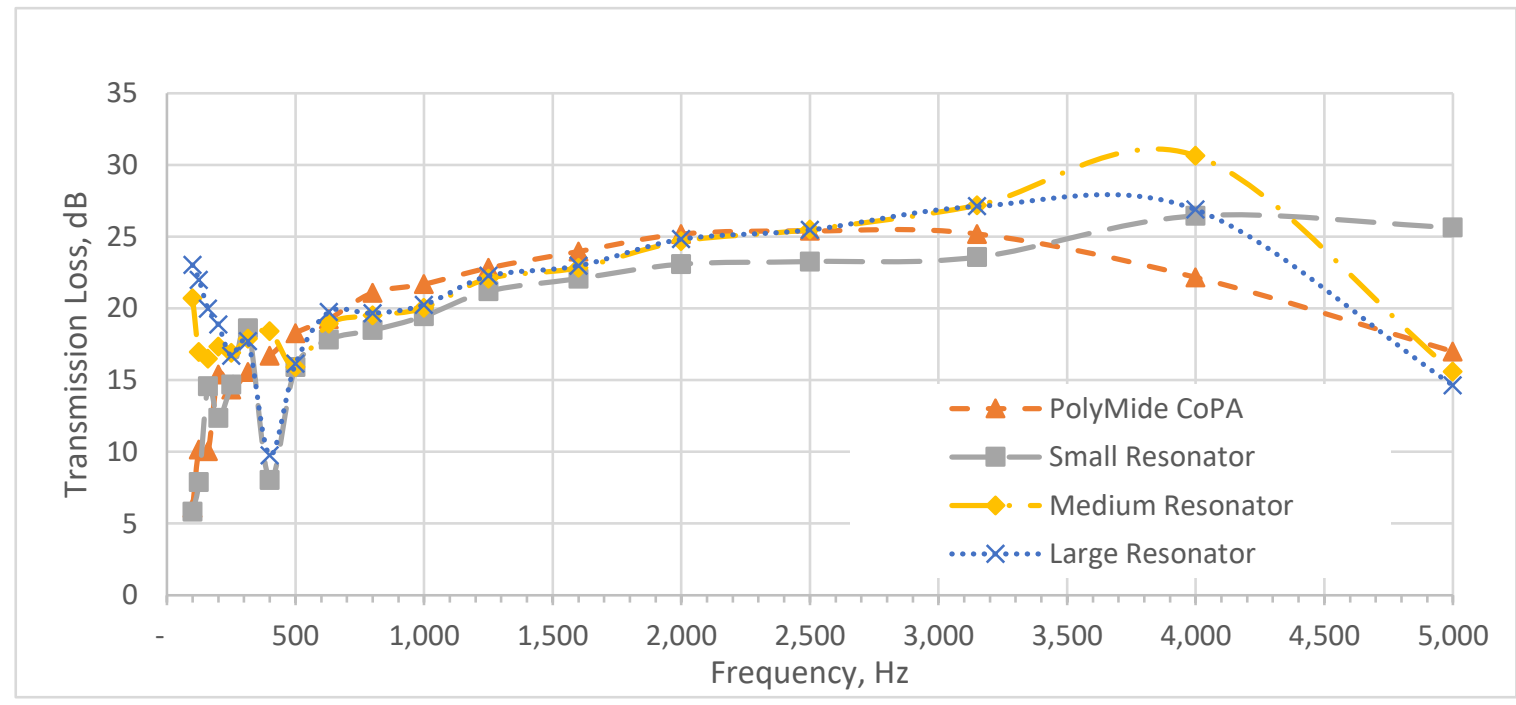

Figure 9. Transmission loss for all unit cell designs made out of PolyMide CoPA

Figure 10 shows the transmission loss values of all unit cell designs manufactured out of PLA. The results show that a local peak, with values of around $23 \mathrm{~dB}-26 \mathrm{~dB}$, was introduced at 4,000 Hz. This peak was reduced from $26 \mathrm{~dB}$ to $23 \mathrm{~dB}$ as the resonator size was increased. Significant reduction was observed as the resonator size was increased from medium to large when compared to when the resonator size was increased from small to medium. Finally, for frequencies between $500 \mathrm{~Hz}-2,000 \mathrm{~Hz}$ the introduction of a resonator and recess reduces the transmission loss when compared to the solid circular prism. The transmission loss values were also observed to be higher when compared to the values obtained from the solid circular prism.

The results from Figures 9 and 10 indicates that the introduction of the resonator within the design serves to principally introduce a peak in the transmission values at around $4,000 \mathrm{~Hz}$. This peak value however was found to be dependent on the size of the resonator, decreasing as the resonator size was increased. For other frequencies, the introduction of the 
resonator in the design did not significantly impact the transmission loss values. These values were in general either higher or lower by around $2 \mathrm{~dB}$ from the transmission values of the material. Changes to the stop band are only observed for frequencies above $3,000 \mathrm{~Hz}$, whereby the transmission loss values were increased due to the metamaterial design.

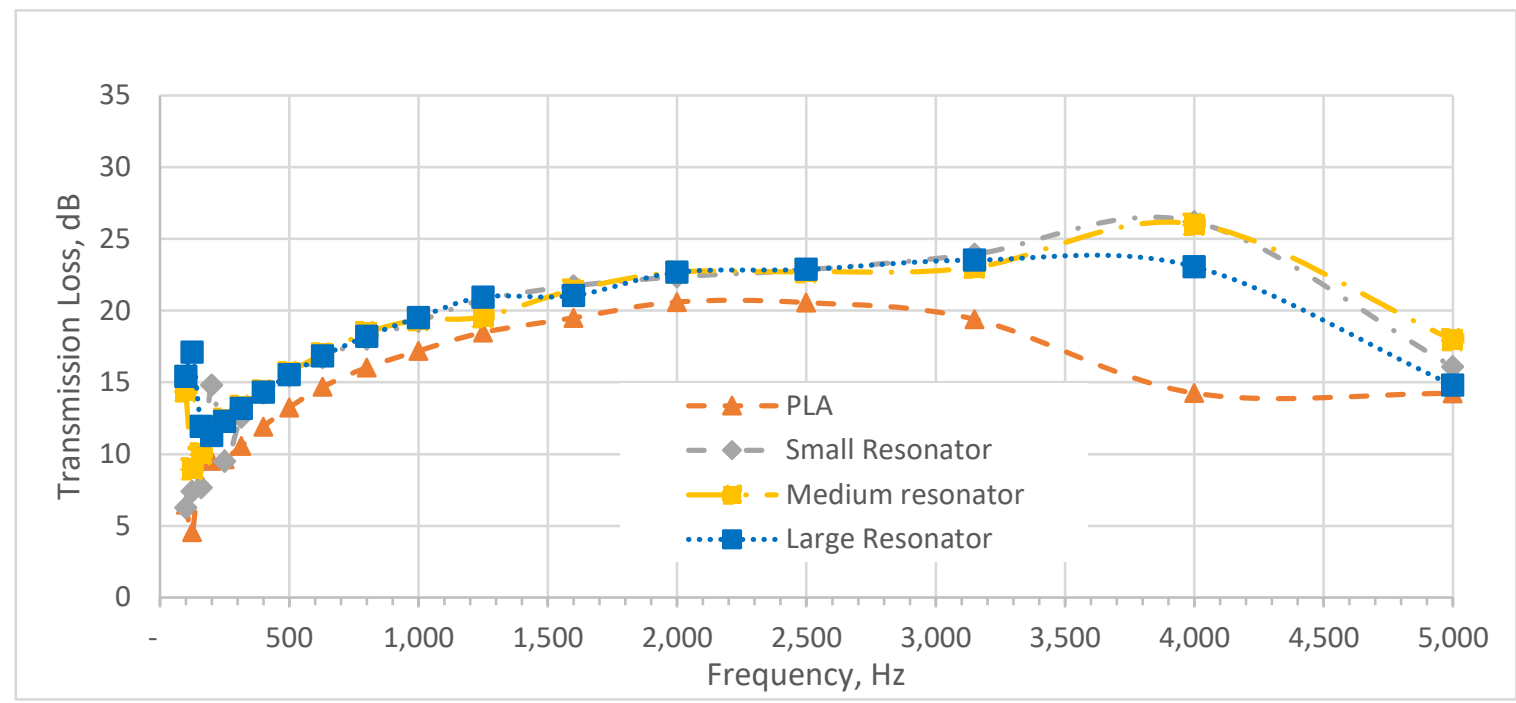

Figure 10. Transmission loss for all unit cell designs made out of PLA

Figure 11 shows a comparison of the absorption coefficient values for the current metamaterial design with the values of a gypsum-based sample (design C-30) manufactured out of 3D printing technology [22]. It is observed that the absorption coefficient values for the metamaterial design are higher for frequencies below 4,500 Hz. For frequencies above $4,500 \mathrm{~Hz}$, the absorption coefficient for design C-30 is higher. This indicates that the design approach, a resonator in a recess manufactured out of a single material, has the potential to increase the absorption coefficient value to lower frequency range. Furthermore, the comparison indicates that the absorption coefficient values obtained are in line with other designs, especially designs manufactured out of a single material using 3D printing technology.

The presence of resonator and recess serves to introduce three peaks in the absorption coefficient values of the material. The peaks were in the $1,000 \mathrm{~Hz}-2,000 \mathrm{~Hz}$, $2,000 \mathrm{~Hz}-3,000 \mathrm{~Hz}$, and $3,500 \mathrm{~Hz}-4,500 \mathrm{~Hz}$ frequency ranges. The first two of these ranges showed similar absorption coefficient values of around $0.20-0.30$. More importantly, the second peak is a reduction in the peak absorption coefficient values of the material. Moreover, these peaks were not significantly impacted by changes to the resonator size. For the peak within the $3,500 \mathrm{~Hz}-4,500 \mathrm{~Hz}$ frequency range, the value of the absorption coefficient varied between 0.15 and 0.30 and showed dependence on the resonator size especially as the resonator size was increased from medium to large.

The inclusion of resonator and recess in the design has significantly less impact on the transmission loss values. For frequencies below $3,000 \mathrm{~Hz}$, the resonator and recess reduce or increases the transmission loss value uniformly by $3 \mathrm{~dB}$ over the frequency range. In addition, the transmission loss values within this frequency range were less affected by the changes to the size of the resonator. However, for frequencies above $3,000 \mathrm{~Hz}$, the resonator and recess introduce a peak transmission loss values, of about $7 \mathrm{~dB}$ at 4,000 $\mathrm{Hz}$. This transmission loss value was affected by changes to the resonator size, being reduced with increasing resonator size. 


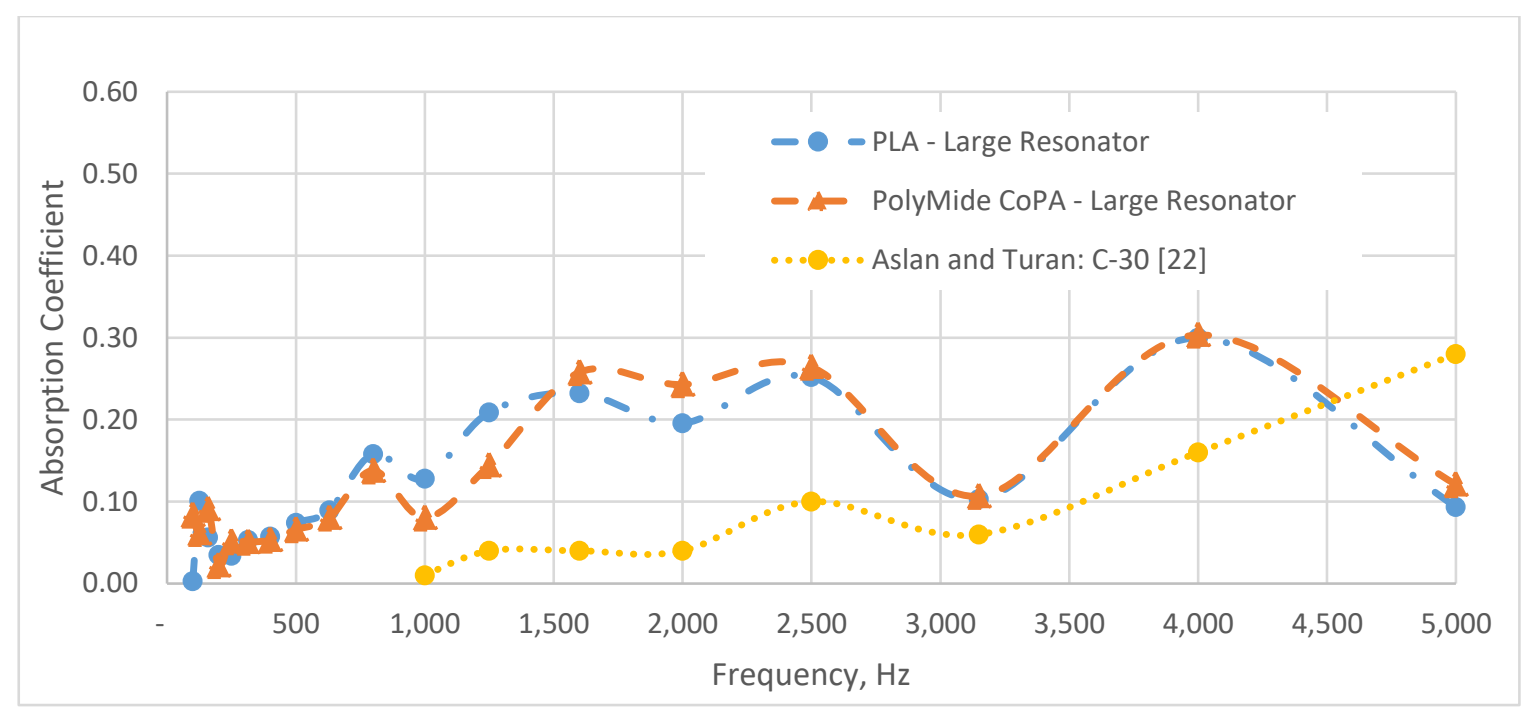

Figure 11. Comparison of absorption coefficient values for large resonator design with gypsum-based design C-30 [22]

The results indicates that the resonator in recess design will reduce the material's peak absorption coefficient values to around 0.25-0.30 regardless of the material used. In addition, two additional peaks will be introduced at a lower and a higher frequencies respectively with the higher frequency peak showing dependence on the resonator size. The impact of the design on the acoustic performance was only observed for frequencies above $1,000 \mathrm{~Hz}$ while very little impact was observed on the absorption coefficient values for frequencies below $1,000 \mathrm{~Hz}$. The results also indicates that the resonator in recess design will increase the peak transmission loss by about $7 \mathrm{~dB}$ at 4,000 $\mathrm{Hz}$ regardless of material used. This peak transmission loss values also shows dependence on the resonator size. Below $3,000 \mathrm{~Hz}$, the design will increase or decrease the transmission loss values by about $3 \mathrm{~dB}$ and is not affected by changes to the resonator size.

\section{Conclusions}

The results indicate that the acoustic metamaterial design, consisting of resonator in a recess made out of a single material, widens the effective frequency range, or stop band, for the absorption coefficient. This widening of the frequency range is through the introduction of additional peaks and the reduction of the peak absorption coefficient values of the material. Changes to the stop band was only observed for frequencies above $1,000 \mathrm{~Hz}$ while very little change was observed for frequencies below $1,000 \mathrm{~Hz}$. This indicates that the current design can be used to widen the stop band for absorption coefficient for frequencies above 1,000 Hz. The results also indicate that the resonator in recess design will increase the peak transmission loss by about $7 \mathrm{~dB}$ for frequencies around $4,000 \mathrm{~Hz}$ with values exhibiting dependence on the resonator size. Below $3,000 \mathrm{~Hz}$, the design will increase or decrease the transmission loss values by about $3 \mathrm{~dB}$ and does not exhibit dependence on the resonator size. Furthermore, the metamaterial design significantly increases the stop band for transmission loss for frequencies above $3,000 \mathrm{~Hz}$.

Together, the study shows that the metamaterial design, consisting of a resonator in a recess, manufactured out of a single material is able to widen the frequency band for the absorption coefficient values for frequencies above $1,000 \mathrm{~Hz}$, with reduction in the peak values. The metamaterial design is also able to increase the stop band for the transmission loss values for frequencies above $3,000 \mathrm{~Hz}$, with significant increase in values within this range. 


\section{Acknowledgement}

This work was supported by Universiti Putra Malaysia (UPM) through the Putra Research Grant (Grant number 9627900 2017).

\section{References}

[1] S. Ornes, "Metamaterials," Proceedings of the National Academy of Sciences of the United States of America, Vol. 110, No. 21, p. 8319, 2013 [Online]. Available: https://www.jstor.org/stable/42656708

[2] A. Hall, E. Calius, G. Dodd, E. Wester, and K. Chan, "Development of locally resonant structures for sonic barriers," Building Acoustics, Vol. 21, No. 3, pp. 199-220, 2014. doi: 10.1260/1351-010x.21.3.199

[3] L.Y.L. Ang, Y.K. Koh, and H.P. Lee, "Acoustic metamaterials: A potential for cabin noise control in automobiles and armored vehicles," International Journal of Applied Mechanics, Vol. 8, No. 5, 2016. doi: 10.1142/s1758825116500721

[4] X. Yu, Z. Lu, T. Liu, J. Zhu, and F. Cui, "Sound transmission through a periodic acoustic metamaterial grating," Journal of Sound and Vibration, Vol. 449, pp. 140-156, 2019. doi: 10.1016/j.jsv.2019.02.042

[5] D.P. Jena, J. Dandsena, and V.G. Jayakumari, "Demonstration of effective acoustic properties of different configurations of Helmholtz resonators," Applied Acoustics, Vol. 155, pp. 371-382, 2019. doi: 10.1016/j.apacoust.2019.06.004

[6] H.J. Rice, J. Kennedy, P. Göransson, L. Dowling, and D. Trimble, "Design of a Kelvin cell acoustic metamaterial," Journal of Sound and Vibration, Vol. 472, pp. 115-167, 2020. doi: 10.1016/j.jsv.2019.115167

[7] C. Claeys, P. Sas, and W. Desmet, "On the acoustic radiation efficiency of local resonance-based stop band materials," Journal of Sound and Vibration, Vol. 333, No. 14, 2014. doi: 10.1016/j.jsv.2014.03.019

[8] C. Claeys, K. Vergote, P. Sas, and W. Desmet, "On the potential of tuned resonators to obtain low-frequency vibrational stop bands in periodic panels," Journal of Sound and Vibration, Vol. 332, No. 6, 2013. doi: 10.1016/j.jsv.2012.09.047

[9] U. Fano, "Effects of configuration interaction on intensities and phase shifts," Physical Review, Vol. 124, No. 6, 1961. doi: 10.1103/PhysRev.124.1866

[10] H. Peng, P. Pai, and H. Deng, "Acoustic multi-stopband metamaterial plates design for broadband elastic wave absorption and vibration suppression," International Journal of Mechanical Sciences, Vol. 103, pp. 104-114, 2015. doi: 10.1016/j,ijmecsci.2015.08.024

[11] C. Claeys, N. Rocha de Melo Filho, L. Van Belle, E. Deckers, and W. Desmet, "Design and validation of metamaterials for multiple structural stop bands in waveguides," Extreme Mechanics Letters, Vol. 12, pp. 7-22, 2017. doi: 10.1016/j.eml.2016.08.005

[12] Z. Liu, X. Zhang, Y. Mao, Y. Zhu, Z. Yang, C. Chan, and P. Sheng, "Locally resonant sonic materials," Science, Vol. 289, No. 5485, 2000. doi: 10.1126/science.289.5485.1734

[13] F. Langfeldt, J. Riecken, W. Gleine and O. von Estorff, "A membrane-type acoustic metamaterial with adjustable acoustic properties," Journal of Sound and Vibration, Vol. 373, No. Supplement C, pp. 1-18, 2016. doi: 10.1016/j.jsv.2016.03.025

[14] F. Langfeldt, W. Gleine, and O. von Estorff, "Analytical model for low-frequency transmission loss calculation of membranes loaded with arbitrarily shaped masses," Journal of Sound and Vibration, Vol. 349, No. Supplement C, pp. 315-329, 2015. doi: 10.1016/j.jsv.2015.03.037

[15] G. Ma, M. Yang, S. Xiao, Z. Yang, and P. Sheng, "Acoustic metasurface with hybrid resonances," Nature: Materials, Vol. 13, No. 9, pp. 873-878, 2014 . doi: 
10.1038/nmat3994

[16] N. Gao, and Y. Zhang, "A low frequency underwater metastructure composed by helix metal and visocleastic damping rubber," Journal of Vibration and Control, Vol. 25, No. 3, pp. 538-548, 2019. doi: 10.1177/1077546318788446

[17] S. Sang, and E. Sandgren, "Study of two-dimensional acoustic metamaterial based on lattice system," Journal of Vibration Engineering \& Technologies, Vol. 6, pp. 513-521, 2018. doi: 10.1007/s42417-018-0068-6

[18] T.Y. Huang, C. Shen, and Y. Jing, "Membrane- and plate-type acoustic metamaterials," Journal of the Acoustical Society of America, Vol. 139, No. 6, 2016. doi: $10.1121 / 1.4950751$

[19] L.Y.L. Ang, Y.K. Koh, and H.P. Lee, "Plate-type acoustic metamaterials: Evaluation of a large-scale design adopting modularity for customizable acoustical performance," Applied Acoustics, Vol. 149, pp. 156-170, 2019. doi: 10.1016/j.apacoust.2019.01.027

[20] M. Chen, W. Xu, Y. Liu, K. Yan, H. Jian, and Y. Wang, "Band gap and double-negative properties of a star-structured sonic metamaterial," Applied Acoustics, Vol. 139, pp. 235242, 2018. doi: 10.1016/j.apacoust.2018.04.035

[21] J.S. Choi, and G.H. Yoon, "Acoustic metamaterials with combined heterogeneous double-split hollow sphere for noise reduction," Journal of Vibration and Control, Vol. 24, No. 21, pp. 4933-4944, 2018. doi: 10.1177/1077546317739794

[22] R. Aslan, and O. Turan, "Gypsum-based sound absorber produced by 3D printing technology," Applied Acoustics, Vol. 161, pp. 107-162, 2020. doi: 10.1016/j.apacoust.2019.107162

[23] Polymaker, Polymaker PolyMide CoPA - Technical Data Sheet, February 2020. [Online].

Available: https://polymaker.com/Downloads/TDS/PolyMide_\%20CoPA_TDS_V4.pdf. [Accessed: July 2020]

[24] Polymaker, Polymaker PolyLite PLA - Technical Data Sheet, November 2018. [Online]. Available: https://polymaker.com/Downloads/TDS/PolyLite_PLA_TDS_V4.pdf. [Accessed: July 2020]

[25] P.S. Liu, and G.F. Chen, "Characterization Methods: Physical Properties," In Porous Materials, P.S. Liu and G.F. Chen, eds.: Butterworth Heinemann, Boston, United States, pp. 493-532, 2014.

[26] American Society for Testing and Materials (ASTM) International, Standard Test Method for Impedance and Absorption of Acoustical Materials Using A Tube, Two Microphones and A Digital Frequency Analysis System: ASTM E1050-12, West Conshohocken, United States, 2012. doi: 10.1520/E1050-12

[27] Brüel \& Kjær, Technical Review No 1. 2007: Transmission Loss Measurements in a Standing Wave Tube, Vol. 1, Naerum, Denmark, 2007 [Online]. Available: https://www.bksv.com/media/doc/bv0059.pdf [Accessed: July 2020]

[28] M. Vorländer, "Annex: Material Data," In Auralization: Fundamentals of Acoustics, Modelling, Simulation, Algorithms and Acoustic Virtual Reality, Springer-Verlag, Berlin Heidelberg, Germany, pp. 303-318, 2008. 